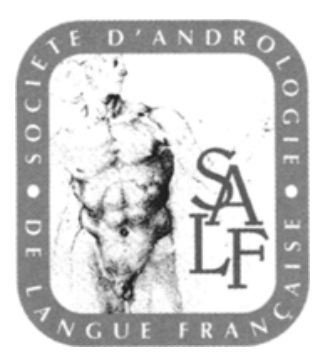

Le dossier comprend trois volets : 1. Présentation du candidat, 2. Projet de recherche et 3. Plan de financement. II doit être accompagné d'une lettre de recommandation du Directeur du Service ou du Laboratoire dans lequel la recherche sera menée.

\section{Présentation du candidat}

- un Curriculum Vitae, décrivant en particulier la situation administrative et financière du candidat. La Société d'Andrologie de Langue Française vous demandera de préciser votre situation financière en juin 2005 avant l'attribution définitive de la bourse.

- Présentation du Service ou du Laboratoire dans lequel la recherche sera conduite, à l'aide d'un organigramme. Précisez le nombre de personnes sur le projet (autres praticiens ou chercheurs, personnel technique) et la répartition du temps consacré au projet pour chaque participant.

- Lettre de soutien à la candidature, rédigée par le Directeur du Service ou du Laboratoire dans lequel la recherche sera conduite.

2. Projet de recherche détaillant les items suivants (10 pages maximum)

- Situation du sujet sur le plan national et international et définition des objectifs du travail proposé.

- Description des méthodologies employées

- choix des techniques

- nombre de sujets, nombre d'échantillons

- évaluation et méthodologie statistique envisagées

- joindre les documents de support si besoin (par exemple support d'enquête)

- Résultats et perspectives attendus

\section{Financement du projet}

- Présentez votre plan de financement, en indiquant la somme demandée et sa répartition entre i) bourse pour le candidat, ii) financement d'équipement et iii) budget de fonctionnement (les candidats peuvent ne pas demander de financement pour l'un ou l'autre des trois items).
- Précisez si vous bénéficiez d'autres financements (acquis ou demandés) pour ce projet. Dans le cas d'un autre financement, veuillez en donner la répartition selon ces mêmes items.

\section{Calendrier}

Date limite de réception des dossiers :

\section{le 31 JANVIER 2005.}

Les dossiers doivent être envoyés à la fois :

- Par courrier de surface, en deux exemplaires, adressé à :

Olivier Rampin, Analyse et Modélisation en Imagerie Biologique, bâtiment 325, Institut National de la

Recherche Agronomique, 78352 Jouy-en-Josas Cedex, France

Par courrier électronique, adressé à :

rampin@jouy.inra.fr

Les candidats seront prévenus des résultats de l'expertise de leur dossier à la fin du mois de juin 2005, par courrier.

\section{${ }^{\star \star \star} \mathrm{A}$ lire avec attention ${ }^{\star \star \star}$}

Le candidat dont le projet aura été financé par la Société d'Andrologie de Langue Française s'engage à :

- Réaliser le travail durant l'année qui suit celle de l'attribution de la bourse

- Mentionner dans les publications qui sont issues de ce travail qu'il a été subventionné (en partie, le cas échéant) par la SALF.

- Rédiger, avec le Directeur du Service ou du Laboratoire, une revue sur le sujet dans Andrologie.

- Enfin présenter oralement le projet au mois de décembre suivant l'attribution de la bourse, à l'occasion du Congrès Annuel de la SALF, et présenter les résultats du travail à l'occasion du Congrès Annuel de la SALF l'année suivante.

Etabli à l'automne 2004. 